\title{
Perioperative treatment of liver transplantation
}

\author{
Shengbin Zhang * Rui Liu, Shipeng Song, Weiqing Wang, Jin Zhao, Xiaoshi Dong \\ The Third Affiliated Hospital of Inner Mongolia Medical University, Hohhot, China
}

Received: June 10, 2012

Accepted: July 20, $2012 \quad$ Online Published: October 15, 2014

DOI: $10.14725 /$ dcc.v1n1p12

URL: http://dx.doi.org/10.14725/dcc.v1n1p12

\begin{abstract}
A case of liver transplantation in the department of general surgery of the third affiliated hospital of Inner Mongolia medical school was collected and analyzed on the basis of diagnosis, physical examination and treatment. Liver transplantation was very complicated, accompanied by various postoperative complications. So this paper aims to share experience of perioperative management of liver transplantation among physicians.
\end{abstract}

Key Words: Liver transplantation, Perioperative treatment, Perioperative management

\section{Medical record}

A 54-year-old male patient with repeated fatigue and anorexia for 6 years and hepatic space-occupying lesions for 5 years was admitted to our hospital on July 20, 2010. He was confirmed to be a carrier with hepatitis B surface antigen-positive after a routine health examination 25 years ago and was untreated. Consequently, the patient was taking routine physical examination yearly hereafter. He was referred to local hospital due to fatigue and anorexia of unexplained cause, accompanied by nausea and loss of appetite in 2004. He received oral administration of lamivudine 100 $\mathrm{mg}$, qd, when he was diagnosed with cirrhosis under ultrasound and CT examination. Follow-up examination showed hepatic space-occupying lesions about $3 \mathrm{~cm}$ in size in 2005 . Eventually he underwent surgical excision (specific type of operation was unknown) in local hospital with a good recovery. The patient had interventional treatment (details unknown) in the local hospital in 2006 and 2009 as hepatic carcinoma reoccurred. Hepatic space-occupying lesions were enlarged to be $7 \mathrm{~cm} \times 6 \mathrm{~cm}$ in size under abdominal ultrasound at present. Her skin and sclera was normal, no symptoms of fever, shiver, abdominal pain and abdominal distension were presented, and no changes in bowl habits since the occurrence of the disease. There was no particularity in his past history, family history or vaccination history.

\section{Physical examination}

Data on physical examination included T $36.4^{\circ} \mathrm{C}, \mathrm{P} 86 / \mathrm{min}$, R 23/min, BP 120/80 mmHg. Though his skin and sclera were without xanthochromia, he was presented with chronic liver disease appearance. No liver palms and spider angiomata and no obvious swelling of all superficial lymph nodes were found. Heart and lungs were normal and abdominal was flat. No gastrointestinal and peristaltic wave and gastric varices of abdominal wall were detected. Abdominal was tender without rebound tenderness and muscle tension. No enlargement of liver, spleen and kidneys beneath the rib was touched. Percussion pain could not be felt at the fifth intercostal space beneath right mid-clavicular line of upper bound of the liver. Shifting dullness was negative and bowl sound was normal. No edema of lower limbs was seen and nervous system was normal.

\section{Laboratory examination}

The results of the examination are as follows. Blood test: $\mathrm{WBC} 3.2 \times 10^{9} / \mathrm{L}, \mathrm{Hb} 130.9 \mathrm{~g} / \mathrm{L}$, PLT $51 \times 10^{9} / \mathrm{L}$.

\footnotetext{
${ }^{*}$ Correspondence: Shengbin Zhang, E-mail: nmgbgyyyy@163.com. Address: Department of General Sugury, The Third Affiliated Hospital of Inner Mongolia Medical University, Hohhot, China
} 
Blood gas analysis: $\mathrm{pH}$ 7.392, $\mathrm{PCO}_{2} 38.5 \mathrm{mmHg}, \mathrm{PO}_{2}$ $66.4 \mathrm{mmHg}, \mathrm{BE}-1.6 \mathrm{mmol} / \mathrm{L}, \mathrm{HCO}_{3}^{-} 22.9 \mathrm{mmol} / \mathrm{L}$, ammonia: $41.1 \mu \mathrm{mmol} / \mathrm{L}$. Infection four items: HBsAg negative, Anti-HCV negative, Anti-HIV negative, Anti-TP negative, $\mathrm{HBcAb}$ positive, potassium $3.92 \mathrm{mmol} / \mathrm{L}$, sodium $141.1 \mathrm{mmol} / \mathrm{L}$, chloride $104.4 \mathrm{mmol} / \mathrm{L}$, Glu $5.26 \mathrm{mmol} / \mathrm{L}$, calcium $2.3 \mathrm{mmol} / \mathrm{L}$. Liver function: ALT $52 \mathrm{U} / \mathrm{L}$, AST $55 \mathrm{U} / \mathrm{L}$, TBIL $29.9 \mu \mathrm{mmol} / \mathrm{L}$, DBIL $10.3 \mu \mathrm{mmol} / \mathrm{L}$, ALB 38 g/L, TP 73.6 g/L, blood coagulation test: PT 15.3 s, PTA $71.5 \%$, APTT 35.4 s, TT 18.8 s, Fbg 2.921 g/L. Ultrasonography confirmed cirrhosis changes at decompensated stage. Changes of liver mesenchymal, hypersplenotrophy, abnormalities echogenicity of right lobe of liver indicated the diagnosis of hepatic carcinoma. Gallbladder wall roughness and the diastolic function of left ventricular were degraded CT scan demonstrated the cirrhosis of the liver. After the surgery and interventional therapy of hepatocellular carcinoma, the condition was getting better. Endoscopy: the esophageal varices and portal hypertensive gastropathy, dodecadactylitis. Chest X-ray: lungs texture was increased.

\section{Primary diagnosis}

(1) Chronic hepatitis B cirrhosis at decompensation stage

- Portal hypertension

- Esophageal varices

(2) Primary hepatic carcinoma

\section{Diagnosis and treatment}

Allograft transplantation was performed in the evening at 10 o'clock on July 30, 2010 and was through a large incision (30 $\mathrm{cm}$ in size) in the upper abdomen. The upper abdominal wall was incised layer by layer. So basic conditions could be seen through the incision, which included: no ascites in abdominal cavity was seen, local parts was closely adhesive, nodules of varying sizes exhibited in the surface of the left and right liver, metastatic nodules were not around, gastrointestinal tract was normal but pancreas was abnormal. Furthermore, the transplant operation can be conceptualized as consisting of the hepatectomy phase, the anhepatic phase, and the postimplantation phase. Numerous anastomoses and sutures, and many disconnections and reconnections of abdominal and hepatic tissue, must be made for the transplant to succeed during hepatectomy phase. Periodontal ligament and adhesive tissue was firstly disconnected and followed by anatomy of the hepatic hilum. Hepatocystic duct and hepatic artery were then cut and ligated. Portal vein was disconnected about $1.3 \mathrm{~cm}$ in diameter, the second hepatic hilum, superior vena cava and inferior vena cava were disconnected respectively. Portal vein and inferior vena cava were blocked, so the native liver was removed. Hemostasis was performed since there was sever wound oozing. Cured

Published by New Century Science Press hepar were placed into the right upper quadrant after the bleeding was stopped. 3-0 wire was used to continuous anastomosis of superior vena cava and inferior vena cava and 5-0 wire was used to anastomose portal vein. Anastomotic stoma should be closely monitored for the prevention of wound oozing. Next came the anhepatic phase for 90 minutes. Hepatic hyperaemia was normal and was soaked in warm slain in order to promote circulation. Hepatic arteries were continuously anastomosed $0.37 \mathrm{~cm}$ in diameter with normal pulse, and wound oozing was presented. Bile duct was anastomosed $0.4 \mathrm{~cm}$ in diameter without biliary leakage around anastomotic stoma. Bile cyst of donor liver was resected with wound oozing, and hemostasis of 12 pad of gauze by compression was followed since upper pole of the right kidney in patients was very adhesive. Drainage tubes were placed respectively. During the surgery, the amount of blood loss was approximately 4,000 $\mathrm{ml}$ and the patient was returned to ICU at 8 o'clock on July 30.

The patient was treated with blood transfusion, hemostasis and pressure rising therapy in that blood pressure was declined gradually with the lowest $68 / 46 \mathrm{mmHg}$. Abdominal fluid drainage was blood and it was continuously increased by $1,100 \mathrm{ml}$. Data on routine blood test revealed WBC 6.0 $\times 10^{9} / \mathrm{L}, \mathrm{Hb} 42 \mathrm{~g} / \mathrm{L}$, PLT $56 \times 10^{9} / \mathrm{L}$. The patient underwent laparotomy surgery for hemostasis at 14 o'clock on July 30. The upper pole of the right kidney was bleeding seriously so that vicryl suture was in use to close it up. There was still regional bleeding after the right kidney was packaged and compressed by optilene mesh. Blooding amount is relatively more at the third hepatic hilum, hence gauzes for compression and suture was done. The patient was returned to ICU thereafter. After the surgery, the patient received anti-infection therapy which consisted of cefoperazone sodium and sulbactam sodium, linezolid and ornidazole. Hemocoagulase for haemostasis, reduced glutathione for liver protection, omeprazole for gastric mucosa due to its acid-suppressive function, albumin support, concentrated red bloodcells for anemia together with plasma transfusion, platelet, cryoprecipitate for blood coagulation improvement were continuously applied. On August 3, the patient recovered completely with normal passage of gas and defecating functions with oral administration of tacrolimus for graft rejection. On the next day, enteral nutritional emulsion of peptison was injected through nasogastric tube. On August 5, fluconazole for graft rejection was added in when sputum culture indicated that yeast-like fungi accounted for $60 \%$. On August 6, about 1,200 $\mathrm{ml}$ of old bloody effusion was extracted under ultrasonography when the bedside examination showed pleural effusion from the right side. At 7 o'clock in the morning on August $8,1,000 \mathrm{ml}$ of bloody liquid was extracted by abdominal drainage. The patient was then sent into operating room again to perform laparotomy which further to find hemostasis in the upper part of the right kidney. Imipenem together with gentamicin was administrated for anti-infection. Gastric tube was removed for 
liquid diet on August 9. The patient could breathe without ventilator on August 10. Follow-up examination on August 17 showed no effusion in pleuroperitoneal cavity under ultrasonography. Routine blood test: WBC $10.9 \times 10^{9} / \mathrm{L}, \mathrm{Hb}$ $93 \mathrm{~g} / \mathrm{L}$, PLT $219 \times 10^{9} / \mathrm{L}$, liver function: ALT $80 \mathrm{U} / \mathrm{L}, \mathrm{AST}$ $62 \mathrm{U} / \mathrm{L}$, TBIL $29 \mu \mathrm{mmol} / \mathrm{L}$, DBIL $19.7 \mu \mathrm{mmol} / \mathrm{L}$, ALB 44.1 $\mathrm{g} / \mathrm{L}, \mathrm{TP} 77.3 \mathrm{~g} / \mathrm{L}$. The patient was discharged hereafter. During follow-up visit of 1 year, neither complication in biliary tract nor tumor recurrence was found. So the accurate diagnosis on the basis of surgical procedures could be concluded: (1) Chronic hepatitis B cirrhosis at decompensation stage, portal hypertension, esophageal varices. (2) Primary hepatic carcinoma. (3) Abdominal haemorrhage and pleural effusion after liver transplantation.

\section{Discussion}

\subsection{Dr. Rui Liu}

Dr. Rui Liu is an attending physician in the Department of General Surgery at the third affiliated hospital of Inner Mongolia Medical University, whose research interests are hepatobiliary surgery and breast disease

Liver is another organ in human body, expect for brain, that has not been fully cognized due to its subtle structure and complicated function. Effective treatment for lesions that had occurred at liver was far from satisfactory. The advent and development of liver transplantation has undoubtedly expanded scope and field of liver disease treatment and promoted progress of hepatic surgery technology. It was the best and effective treatment option for patients with endstage liver disease over the 10 years since the introduction of immunosuppressive drug, improvement of surgical techniques and monitoring. In the United States, there were an estimated 5,000 cases of liver transplantation each year, 3,000 cases annually in Europe, with 1-year survival rate $>$ $90 \%$. Liver transplantation is developing very slowly in our country. After a period of time, research of liver transplantation is so frequent in recent years. Until the end of 2005, it is estimated of 8,000 cases of liver transplantation in China, in which about 3,000 cases were performed only in 2005. ${ }^{[1]}$ Thus, liver transplantation has now been widely acknowledged as the best treatment option for liver disease both in our country and abroad, especially for the disease without effective treatment, for example, end-stage liver disease and some congenital liver metabolic diseases, whose treatment were mainly hepatectomy, are expected to be cured and have a significantly longer lifetime. Primary hepatic carcinoma, one of the highly malignant tuomor, is mostly treated with hepatectomy, regional ablation, intervention, chemotherapy, liver transplantation and so on. Liver transplantation is much preferred for hepatic carcinoma. However, there is no standard criteria for patients' selection since the opinions are verified. The same situation goes for our country. More- over, the prevalence of hepatic carcinoma in China ranks the top around the world as more than half of world's new-onset hepatic carcinoma occurred in our country, ${ }^{[2]}$ in which cases of surgical resection are less than $10 \% .{ }^{[3]}$ Therefore, liver transplantation provides new hope for patients with unresectable liver disease. In 1996, Mazzaferro, et al. proposed a method as selection guidelines for liver transplantation in patients with hepatic carcinoma, known as Milan criteria. ${ }^{[4]}$ It was gradually popularized in the international level and achieved excellent outcome. According to Milan criteria, a single tumor is up to $5 \mathrm{~cm}$ in diameter or 2-3 lesions of up to $3 \mathrm{~cm}$ without macrovascular invasion. ${ }^{[4]}$ But the shortcomings of Milan criteria are as follows: (1) Milan criteria is too restricted. It may preclude a successful transplant for potential patient candidates with hepatic carcinoma. (2) The overall survival rate of patients with liver transplantation within Msilan criteria and those with surgical resection is nearly the same. (3) Some important factors which highly impact the prognosis of liver transplantation for hepatic carcinoma, such as the degree of liver disease, backgrounds of hepatitis, invasion of vascular, metastasis of lymph node were not involved in Milan criteria. Recent several proposals have been made to expand the Milan criteria. ${ }^{[5]}$ Shanghai Fudan criteria that proposed by Jia Fan, et al. ${ }^{[6]}$ in 2006 is widely adopted in China at present. According to the criteria, single tumor $\leq 9 \mathrm{~cm}$ in diameter, multiple tumors $\leq 3$ and the largest tumor $\leq 5 \mathrm{~cm}$ in diameter, the overall diameter of all the tumors $\leq 9 \mathrm{~cm}$ in diameter without major vascular invasion and lymph node metastasis.

The patient, who was diagnosed with primary hepatic carcinoma with tumor of $7 \mathrm{~cm} \times 6 \mathrm{~cm}$ in size, fulfilled Shanghai Fudan criteria.

\subsection{Dr. Shengbin Zhang}

Dr. Shengbin Zhang is a director in the Department of General Surgery at the third affiliated hospital of Inner Mongolia Medical University, whose research interests are liver \& gallbladder diseases and organ transplantation.

Liver transplantation is the only effective treatment method in patients with end-stage liver disease accompanied by many surgical complications. ${ }^{[7]}$ Intraperitoneal hemorrhage is one of the most frequent complications ${ }^{[8]}$ with incident rate of $4.9 \%-13.04 \% .{ }^{[9,10]}$ It usually occurs within 48 hours in the early postoperative period after liver transplantation, whose clinical manifestation are rapid heart rate, lower blood pressure, reduction of urine as well as the decrease of hemoglobin and haematocrit. Postoperative ultrasonography, characterized with lower price, non-invasive quality and convenient bedside examination, serves as a useful tool in detecting effusion in peritoneal cavity or around the liver and massive blood clots. It is of highest value amongst auxiliary examinations in the diagnosis and treatment of intraperitoneal hemorrhage after liver transplantation. There- 
fore, postoperative monitoring of vital signs, the changes on the specific volume of hemoglobin and haematocrit coupled with ultrasound-guided abdominal paracentesis provide primary predictor of accurate diagnosis. Coagulation disorders is a primary reason that accounted for early intraperitoneal cavity after the surgery. It is the main cause of wound errhysis and vascular hemorrhage, especially of intraperitoneal hemorrhage, within 24 hours after the surgery. Patients treated with liver transplantation, particularly for patients with advanced cirrhosis, were presented with preoperative hepatosis, which may lead to obviously insufficient anticoagulant protein synthesis of AT, HCIIPC, PS and so on. Moreover, the decrease of PAI- 1 and $\alpha 2$-antiplasmin together with the rise of tPA result in the significant increase of fibrinolytic activity. Patients with cirrhosis of decompensation stage suffer from hypersplenism of different levels and blood platelet reduction due to too much damage, which lead to coagulation disorders. Secondly, that factors that liver transplantation normally claims a long period together with blood loss during the surgery requires large inventory of blood products and plenty of liquids. The shortage of blood platelet and blood coagulation factor promotes a large consumption of coagulation materials. The use of heparin during anhepatic phase is another factor that contributes to coagulation disorders. The complication generally occurs within 24 hours hereafter the surgery due to shortage of blood coagulation factors and diagnostic fluid infusion. It could be resolved with timely replenish of blood platelet, blood coagulation factor and prothrombin. ${ }^{[11]}$

\subsection{Dr. Shipeng Song}

Dr. Shipeng Song is an associate director in the Department of General Surgery at the third affiliated hospital of Inner Mongolia Medical University, whose research interests are minimally invasive surgery and organ transplants.

The following surgical factor is another reason that accounted for intraperitoneal hemorrhage in the early stage after liver transplantation. (1) Hemorrhage of recipient's vena cava and branches is mainly due to ligation structure detachment of short hepatic vein or perforator vein. (2) Hemorrhage of hepatic artery anastomosis is commonly seen of vascular anastomosis hemorrhage. Hepatic artery anastomosis has a tendency of spasm during the surgery, and hemorrhagic spot is not easy to be found. Moreover, hemorrhage of hepatic artery anastomosis is usually with a larger bleeding amount after the surgery when the angiospasm was relieved. Therefore, factors that drive hepatic artery spasm should be paid more attention when connecting hepatic artery, such as too much disconnection, clamp of hepatic artery and so on. When the connection was constructed, papaverine or lidocaine should be employed for the prevention of hepatic artery spasm, and the procedure that distal end of anastomotic stoma was clamped by non-traumatic forceps should be followed to confirm whether anastomotic stoma was still bleeding. (3) Wound hemorrhage from right adrenal gland is because of its close connection with hepatic artery, so it could easily lead to wound bleeding during the process of disconnect them. (4) Vascular hemorrhage of donor liver, such as hemorrhage of ligamenta triangulare sinistrum and ligamentum hepatogastricum, is usually caused by unskillful ligation. Hence, examination of uncompleted vessel ligation, especially of inferior vena cava wall, ligamenta triangulare sinistrum and ligamentum hepatogastricum, is necessary. Liver capsule and laceration of liver parenchyma should be repaired with more attention. (5) Hemorrhage of donor liver biopsy. Solid ligation of donor liver biopsy is essential and atraumatic suture may be in use if necessary. (6) Incision hemorrhage of abdominal wall artery. Use of electrotome in the abdomen during the surgery, electrocoagulation of abdominal wall artery and postoperative eschar shedding are much associated with hemorrhage.

Exudate from abdominal infection, pus and bile leakage are proved to corrode vessels (especially hepatic arteries and veins), which is the main factor involved in postoperative hemorrhage. Vigorous Gastrointestinal motility such as dysemesia and heavy vomiting during recovering phase may lead to anstomotic site dehiscence of hepatic arteries, hepatic veins and bile duct, which would consequently result in a substantial amount of bleeding.

We believe that prevention of postoperative abdominal bleeding is of significance for patients with liver transplantation to sail through perioperative period. (1) Treatment for patients with preoperative, intraoperative and postoperative coagulation disorders. PT and APTT is usually extended in patients with severe cirrhosis and hepatic failure. Plenty of fresh plasma, blood platelet, contractinogen and prothrombin complex are required due to the reduction of contractinogen and blood platelet. Repeated examination of coagulation function and targeted correction of coagulation disorders are necessary for the decrease of blood oozing from the wound surface. Prothrombin complex and contractinogen should be supplied when PT $>20$ s, APTT $>$ 60 s since blood platelet (optimal amount of $50 \times 10^{9} / \mathrm{L}-100$ $\times 10^{9} / \mathrm{L}^{[12]}$ ) plays an important role in coagulation function. (2) Tight ligation of short hepatic vein or perforator vein together with vessels between right adrenal vein and abdominal muscle is required. (3) Ligament triangular sinistrum, ligamentum hepatogastricum and cystic artery, particularly for liver biopsy site should be ligated skillfully when backtable was being treated. Prolene suture is applicable for ligation if necessary. (4) Prevention and control of intraabdominal infections and biliary leakage. It would be better for patients with preoperative intra-abdominal infection to control the infection prior to liver transplantation. The infection could be controlled by proper use of antibiotics given that the patient had preoperative infection. It must be ensured that drainage tubes are unobstructed. Peritoneal 
lavage could be constructed under strict aseptic condition. (5) During the patient's recovery phase of perioperative period, he needs to avoid vomiting violently for gastrointestinal disorders prevention. The patient in this case was found severe bleeding on the upper pole of the right kidney in the secondary surgical exploration and was timely treated.

\subsection{Dr. Weiqing Wang}

Dr. Weiqing Wang is an associate director in the Department of Intensive Care Unit at the third affiliated hospital of Inner Mongolia Medical University, whose research interests are respiratory and critical care medicine.

Pleural effusion is the most frequent complication after liver transplantation. ${ }^{[13]}$ Long-term untreated pleural effusion in the early postoperative period may eventually lead to severe pulmonary and extrapulmonary complications. While, the pathogenesis of pleural effusion remains unspecific, are related with the following factors. (1) The direct damage caused by the surgery. It has been proved that the impact of liver transplantation on right diaphragm is more severe than that on its counterparts. ${ }^{[14]}$ (2) Hypoproteinemia. Patients are usually presented with hypoproteinemia before the surgery. Blood loss, massive blood transfusion and fluid infusion would further aggravate hypoproteinemia, which only to result in pleural effusion. Pleural effusion, on the contrary, would lead to great loss of protein. On the other hand, the donor liver's limited protein synthesis also contributes to hypoproteinemia, meanwhile hypoproteinemia might facilitate peritoneal effusion, which formulates vicious circulation. While, clinical observation demonstrates that peritoneal effusion could be absorbed in the abdomen by continuously supplement of albumin. (3) Inflammationinduced stimulation before and after the surgery. Most patients were with preoperative malnutrition, deterioration of pulmonary functions, hence they were afraid to cough forcefully after the surgery, with expansion of functional residual air cavity and large dose of immunosuppressive agents leading to pleural effusion caused by pulmonary infection. Reoperation will further increase postoperative diaphragmatic injury, which brought about regional inflammation. Pleural effusion aroused from the mentioned reasons is much of exudate. Previous studies show that dysfunction of vital organs, systemic failure and infection are largely associated with delayed extubation. ${ }^{[15]}$ Tracheal intubation and prolonged mechanical ventilation would weaken cough reflex and ciliary movement, diminish mucosal barrier function, which meanwhile create a method for germ invasion through the tube. ${ }^{[16]}$ There is a casual relationship between pulmonary infection and prolonged presence of pleural effusion.

In general, it is easier for the diagnosis of pleural effusion by X-ray and ultrasonography examination. X-ray features highly suggestive of the symptom are blunted, fuzzy and un- clear costophrenic angle. Its evaluation of pleural effusion is not specific enough and sometimes a small amount could not be displayed. However, the advantage of X-ray lies in the fact that the condition of lungs could be achieved. Ultrasonography examination is an accurate approach for condition of fluid areas even if the amount is small. A precise evaluation of amount of pleural effusion could be expected based on the examination. X-ray examinations and/or ultrasonography should be performed for more information regarding lung condition. The most simple and effective method is deep vein tube placement in the chest guided by ultrasonography, which is proposed to extract pleural fluid one time every day, and should be removed until the absence of the fluid. Deep vein tube placement in the chest is very easy to operate since the tube is soft with little irritant effect. The tube could be placed at the bottom of the chest for a complete drainage since it could be kept for long time to extract repeatedly, which is useful for better clinical effects. On the sixth postoperative day, the patient was found right pleural effusion by chest radiograph, and old blood liquid about 1,200 $\mathrm{ml}$ was extracted by B-mode ultrasound guided deep vein tube. There was no pleural effusion on the next day.

\subsection{Dr. Jin Zhao}

Dr. Jin Zhao is an associate director in the Department of General Surgery at the third affiliated hospital of Inner Mongolia Medical University, whose research interests are gastrointestinal disorders and organ transplant.

Liver transplantation is now widely recognized as an effective treatment option for advanced liver disease. A number of previous studies have found that malnutrition could apparently increase the rate of complication and mortality for patients undergoing liver transplantation. ${ }^{[17,18]}$ The majority of patients who received liver transplantation had been diagnosed with cirrhosis. They were presented with severe malnutrition before the surgery due to sugar, fat, protein metabolism caused by hepatic dysfunction and its long duration. ${ }^{[19]}$ Their bodies were in a high catabolism and metabolic disorders because of the postoperative wound, stress, using of hormones and immunosuppressive agents, etc. Nutrition metabolism wasn't functioning well, usually known as metabolic disorders, in the earlier stage after the surgery because of the impact of warm ischemia, cold ischemia and reperfusion during the surgery. Its clinical manifestations included constricted utilization of glucose, abnormity of sugar tolerance, lower level of hepatic protein synthesis, and increase of decomposition, which may aggravate malnutrition, wound healing difficulties, decline of body resistance as well as the occurrence of complications.

Blocking time of portal vein is associated with blood stasis degrees in gastrointestinal tract, swelling of gastrointestinal mucosa and mucosal injury. Nutrition-support ther- 
apy is especially required due to preoperative malnutrition, surgical stress and large dose of immunosuppressive regimens. Protein metabolism increased significantly, which could be proved by the examination of urea nitrogen in urine. Therefore, protein $(1.5 \mathrm{~g} / \mathrm{kg} . \mathrm{d}-2.0 \mathrm{~g} / \mathrm{kg} . \mathrm{d})$ was proposed to be given for the great loss of urea nitrogen in the earlier stage after the surgery. Quantity of heat, approximately $120 \%-130 \%$ times more than basal energy expenditure, is demanded in the absence of complications. Enteral nutrition has advantages over parenteral nutrition early postoperative period expect the fact that the patient had gastrointestinal dysfunction and could not tolerate the procedure. It is reported that enteral nutrition could significantly lessen incidence of postoperative infection of liver transplantation, and its risk of metabolic complications is lower than that of parenteral nutrition. Besides that, peritoneal drainage of abdominal cavity, the loss of gastrointestinal fluids and excessive fluid during this period might usually lead to electrolyte imbalance. So the use of diuretics should be closely monitored and supplied in that it may arouse gradual loss of potassium, sodium and magnesium in the serum. ${ }^{[20]}$ On the fourth postoperative day, gastric tube peptison feeding was performed to prevent electrolyte imbalance and bacterial translocation in patient's recovering.

\subsection{Dr. Xiaoshi Dong}

Dr. Xiaoshi Dong is a director in the Department of Intensive care unit at the third affiliated hospital of Inner Mongolia Medical University, whose research interests are critical care medicine.

Pulmonary infection is the most common complication that occurred after liver transplantation, with incidence rate of $22.8 \% .^{[21]}$ Its onset is subtle, characterized by rapid progression without remarkable clinical manifestations. Though chest X-ray and computerized tomography scan were primary predictors to determine the diagnosis of pulmonary infection, the diagnosis of pathogens is relatively poor. $\mathrm{Pa}-$ tients with underlying pulmonary infection after liver transplantation should be treated with antibiotics and antifungal drug prior to the specific results of laboratory test. ${ }^{[22]}$ The key factors involve in selecting the drugs are gram-negative bacilli and gram-positive cocci, so imipenem, meropenem, vancomycin and so on are the best treatment option. If the patients were suspected with fungal infections, fluconazole, itraconazole, caspofungin and amphotericin B should be given for treatment. Besides that, an adequate dose regime of hyperimmune globulin and ganciclovir should be given to treat proven or suspected viral infection. Over-reliance on chest X-ray scan and pathogens examination may lead to a delay in early diagnosis and treatment with best outcome for the patients. The increase of pulmonary infection incidence and mortality is largely associated with delayed effective antibiotic therapy. Therefore, careful observation, sample preservation and test for identification of pothogens are required for early diagnosis to prevent the progression of pulmonary infection. Repeated tests of pathogenic microorganisms from different parts seem essential for early identification of pathogens and targeted administration since sever pulmonary infection is the presentation of mixed infection of varying pathogens. ${ }^{[23]}$ The main clinical manifestations caused by pathogenic bacteria often obscure the clinical manifestations of other pathogens. Besides that, many non-specific symptoms would easily confound with graft injection and surgical complications. That is, clinical manifestations as diagnostic foundation alone or empiric treatment of pulmonary infection would postpone the best treatment of the disease. Recent years saw an increase in the proportion of fungal infection, so more attention should be paid to its treatment. Moderate, progressive, collaborative and intermittent application of antifungal agents ${ }^{[24]}$ was suggested to be performed. Mycobacterium tuberculosis is very rare amongst patients who underwent liver transplantation, characterized with high incidence and mortality of extrapulmonary and disseminated tuberculosis.

Consequently, the use of antibiotics should be suspended when the infection was controlled to prevent the occurrence of second infection. The patient in this case was treated with proper antibiotics for postoperative pulmonary infection prevention, which turned out to be successful. Liver transplantation has been regarded as the most effective treatment option for advanced liver disease, especially for patients with liver cancer. The surgery, however, is very complicated accompanied with postoperative complications, so perioperative management should be strengthened for higher success rate. The patient was not accompanied with pulmonary infection after the surgery due to timely application of effective antibiotics. Because of the closely monitoring of vital sign and peritoneal fluid drainage, abdominal haemorrhage and pleural fluid had been discovered and treated on time.

\section{References}

[1] Xiaoping Chen. Liver transplantation for liver surgery. Chinese Journal Of Surgery. 2007; 45(9): 580-583.

[2] Daiming Fan, Guohong Han, Xiangjie Meng. Improve the Curative Effect of Interventional Treatment for Hepatocellular Carcinoma. Chinese Journal Of Digestion. 2006; 26(5): 289-290.

[3] Mengchao Wu, Han Chen, Feng Shen, et al. Influence of Minimally invasive in the surgical treatment of hepatocellular carcinoma. Chi- nese Journal Of Minimally Invasive Surgery. 2002; 2(2): 69-70.

[4] Mazzaferro V, Regalia E, Doci R, et al. Liver transplantation for the treatment of small hepatocellular carcinomas in patients with cirrhosis. N Engl J Med. 1996; 334(11): 693-699. PMid:8594428. http://dx.doi .org/10.1056/NEJM199603143341104

[5] GuJin, Internal Medicine progress of Modern clinical medicine tumour book $[\mathrm{M}]$. Beijing: Science and Technology Literature Publishing House; 2006. 17-20p.

[6] Jia Fan, Jian Zhou, Shu Xu, et al. Select the indications for liver 
transplantation hepatocellular carcinoma: Shanghai Fudan Criteria. Chinese Medical Journal. 2006; 86(18): 1227-1231.

[7] Prabhakaran K, Patankar JZ, Quak SH. Surgical complications and outcome of pediatric liver transplantation: The Singapore experience. Pediatr Surg Int. 2005; 1(8): 609-614. PMid:16049712. http://dx.doi.org/10.1007/s00383-005-1482-x

[8] Mueller AR, Platz KP, Kremer B. Early postoperative complications following liver transplantation. Best Pract ResClin Gastroenterol. 2004; 18(5): 881-900. PMid:15494284. http://dx.doi. org/10.1016/j.bpg.2004.07.004

[9] Yafu Wu, Yudong Qiu, Chunping Jiang, et al. Prevention and Treatment of Early Postoperative intraperitoneal hemorrhage after liver transplantation. Journal of Hepatobiliary Surgery. 2007; 15(1): 1820.

[10] Jianghua Ran, Jing Liu, Shengning Zhang, et al. Activity of intraperitoneal hemorrhage after Liver Transplantation prevention and treatment. China Journal Of Modern Medicine. 2009; 19(5): 751753.

[11] Lvnan Yan. Liver transplantation for hepatic benign disease. Chinese Journal Of Bases And Clinics In General Surgery. 2003; 10(4): 321-322.

[12] Jianguo Xie, Lvnan Yan. Role of liver transplantation in treatment of portal hypertension and Perioperative treatment. Chinese Journal Of General Surgery. 2005; 114(6): 404-406.

[13] Singh N, Gayowski TW, agener MM, et al. Pulmonary infi-ltrates in liver transplant recipients in the intensive care unit. Transplantation. 1999; 67: 1138. PMid:10232564. http://dx.doi.org/10. 1097/00007890-199904270-00009

[14] AFESSA B. Pulmonary complications of orthotopic liver transplantation. Mayo Clin Proc. 1993; 68: 427-428.
[15] Shusen Zheng, Anwei Lu, Yanbo Liang, et al. Causes and Countermeasures of respiratory complications after liver transplantation. Journal of Zhejiang University. 2004; 33(2): 170-173.

[16] Jianan Ren. Characteristics and treatment of perioperative respiratory tract bacterial infection. Chinese Journal of Practical Surgery. 2004; (24): 144-145.

[17] Yousheng Li, Jieshou Li. Nutritional support of patients with organ transplants. Parenteral \& enternal nutrition. 2003; 10(2): 1111.

[18] Zaohan Wu. Practical clinical nutrition therapy. ShangHai: Shanghai Science and Technology Press; 2001. 3621p.

[19] Ke Zhang, Huifen Wang, Wenbing Sun. Enteral nutrition support and on malnutrition liver cirrhosis. Foreign Medical Sciences in fascicles. 2002; 29(11): 468-4691.

[20] Sanchez A, Aranda-Michel J. Nutrition for the liver transplant patient. Liver Transpl. 2006; 12: 1310-1316. PMid:16933224. http: //dx.doi.org/10.1002/lt.20894

[21] Shen YH, Fan J, Zhou J, et al. Pulmonary infection and its risk factors after orthotopic liver transplantation. Zhonghua Gan Zang Bing Za Zhi. 2007; 15(11): 833-936.

[22] Ruipeng Hou, Binyi Shi, Guosheng Du, et al. In-situ Clinical Analysis of 8 cases of pulmonary infection after Liver Transplantation. Academic journal of pla postgraduat medical school. 2008; 29(1): 46-47.

[23] Changjie Cai, Guihua Chen, Xiangdong Guan. et al. Epidemiological analysis of bacterial infection after liver transplantation. Chinese Journal of Practical Surgery. 2003; 23(3): 163-164.

[24] Qiyuan Lin, Jiayin Yang, Lvnan Yan, et al. Early etiology of patients after Liver Transplantation and Clinical study of infection. Parasitoses and Infectious Disecses. 2003; 1(2): 55-58. 